\title{
Upgrade project and plans for the ATLAS detector and first level trigger.
}

\section{Domenico della Volpe*}

On behalf of the ATLAS Collaboration

I.N.F.N. \& Università degli Studi di Napoli 'Federico II',

Dipartimento di Scienze Fisiche,

Via Cintia

80126 - Napoli,

E-mail: Domenico.Della.Volpe@cern.ch, dellavolpe@na.infn.it

In the coming years, the LHC complex will be upgraded to extend the physics potential of the experiments. The average luminosity will be increased by a factor 5 to 10 above the original design one. The planned upgrades require, among other detector and DAQ system improvements, a significant higher selectivity of the trigger system, to cope with the increased radiation level and particle rates.

In this paper we describe the changes to the ATLAS first level of trigger system currently under study. The calorimetry based trigger detectors will improve their selectivity by benefiting from the increased granularity available at the trigger level, which will allow for a higher energy resolution. In the muon detector, the momentum resolution of the trigger can be improved by using the precision muon tracking detectors. The use of the inner tracking system in the lower levels of the trigger selection is also discussed.

36th International Conference on High Energy Physics

4-11 July 2012

Melbourne, Australia

\footnotetext{
*Speaker.
} 


\section{Introduction}

The Large Hadron Collider (LHC) represents a unique possibility to explore unprecedented energy frontier in the search of new physics and in the precision measurements of known phenomena. The collider operations, begun in 2009 , are expected to extend through different phases up to 2021, delivering an integrated luminosity of more then $300 \mathrm{fb}^{-1}[1]$,

Starting from 2019 , the peak luminosity will reach $2 \div 3 \times 10^{34} \mathrm{~cm}^{-2} \mathrm{~s}^{-1}$, which is beyond the design specifications of the ATLAS experiment[2] that thus needs to upgrade some of the components. This period is often referred to as Phase-I. With a further upgrade of the collider (HL-LHC) and its injection line, the instantaneous luminosity should reach $5 \div 7 \times 10^{34} \mathrm{~cm}^{-2} \mathrm{~s}^{-1}$ allowing for delivering a total integrated luminosity of around $3,000 \mathrm{fb}^{-1}$ in 10 years (Phase-II).

While it is quite difficult to predict what the physics scenario will be in the next future, the ATLAS upgrades shall maintain the same physics potential for probing the origin of the electroweak symmetry breaking. Particular emphasis will be given to the Higgs boson couplings measurement, or if not confirmed, to WW boson scattering measurements.

In order to maintain its rich and varied physics program, the ATLAS detector and trigger system must perform excellently down to the GeV energy scale in a much harsher and crowded environment as HL-LHC will be.

In fact, the increase in luminosity will come at some costs: the average number of interactions per beam crossing will increase, ranging from 80 during Phase-I up to 200 for Phase-II. Correspondingly the detectors occupancy will increase degrading the system performance. So for example, the effect of piling-up many events will worsen the calorimeters energy resolution as well as the pattern and track reconstruction efficientcy.

Moreover, during 10 years, in 2021, the detectors will absorb huge radiation doses. For some components, it will be either near or beyond the design limit and they will need to undergo major refurbishing or complete replacement.

In this paper we will concentrate in particular on the evolution and the upgrade of the first level, among three, of the ATLAS trigger, elsewhere described[3, 4].

\section{The Level-1 trigger challenge at high luminosity}

The present Level-1 trigger rates, scaled at luminosity higher than the design value, would exceed its design value of $100 \mathrm{kHz}$.

The trigger rate will increase not only for the corresponding physics process, but also because the random coincidences faking the trigger will inevitably increase with the higher occupancy and worst resolutions.

More refined and complex algorithms can maintain high efficiency while increasing the purity of the selected physics objects, and then keep the rates at a reasonable level.

The main strategy of the ATLAS systems upgrades foresees to increase the trigger rejection power by using higher granularity where possible, adding new detectors or using detectors information that are currently not used for triggering.

Then the Level-1 system will need to handle more information requiring more processing power and a longer time to both read-out and elaborate the trigger decision. 
The increase of the trigger latency is limited by the depth to the data storage buffers, which is $2.5 \mu \mathrm{s}$ in the current system.

In order to handle both the increased rates and the latencies in Phase-II, most of the present electronics would be changed. Unfortunately, the electronics of the precision chambers in the inner part of the Muon spectrometer cannot be replaced without major dissembling. Recent studies show that, at the expected hit tube rate of $100 \mathrm{kHz} / \mathrm{cm}^{2}$ in Phase-II, the present buffer length allows to absorb a latency up to $20 \mu$ s and the electronics can stand up to $200 \mathrm{kHz}$. The possibility of changing this electronics is anyhow under study.

\section{The Level-1 Muon trigger upgrades}

The current Level-1 muon system has shown unexpectedly high rates of fake triggers in the end-cap region $(|\eta|>1.05)$ of the muon spectrometer (cfr. Fig. 1), where the highest cavern background flux is expected. Cavern background is mainly due to low energy photons and neutrons generated by beam protons hitting the beam pipe, which cause high level of random hits in the detectors. Charged particles can also escape and penetrate the layers of the detectors, producing track segments. The expected rate of muons with transverse momentum greater than $20 \mathrm{GeV} / \mathrm{c}$ at $\sqrt{s}=14 \mathrm{TeV}$ and instantaneous luminosity $3 \times 10^{34} \mathrm{~cm}^{-2} \mathrm{~s}^{-1}$, is $60 \mathrm{kHz}$ out of the $100 \mathrm{kHz}$ of the total Level-1 rate.

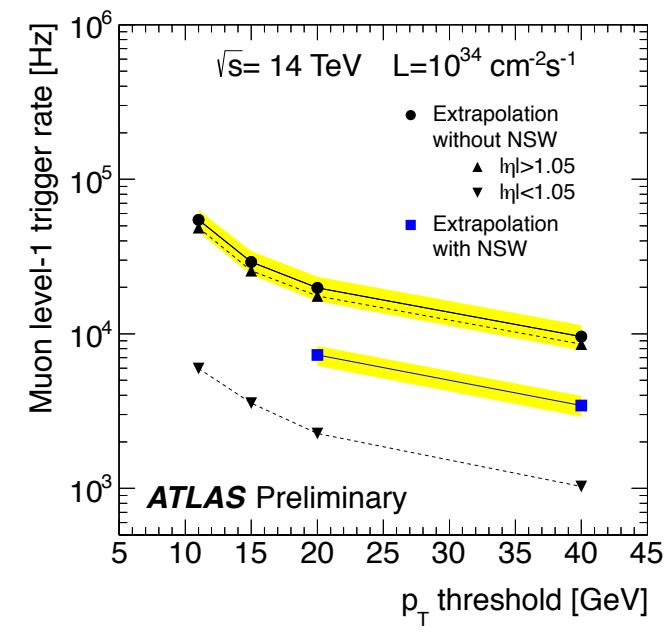

Figure 1: Estimation of the ATLAS muon Level1 trigger rates extrapolated at $\sqrt{s}=14 \mathrm{TeV}$ for $\mathscr{L}=$ $10^{34} \mathrm{~cm}^{-2} \mathrm{~s}^{-1}$ as a function of the $p_{T}$ threshold. The effect of the NSW is shown for $p_{T} \geq 20 \mathrm{GeV}$ by the blue squared points

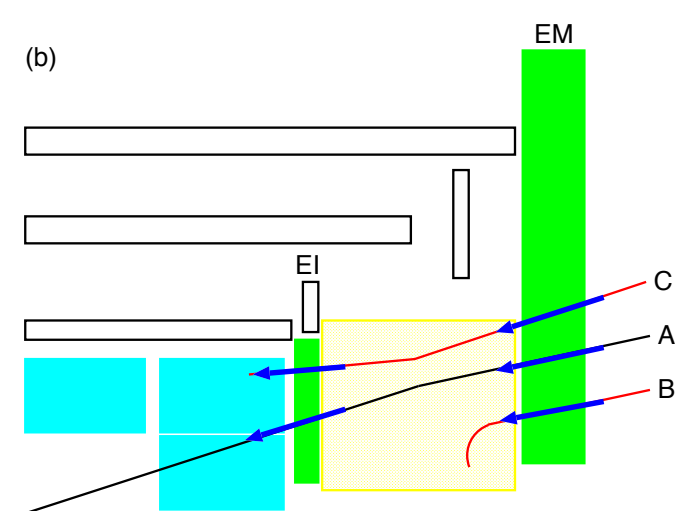

Figure 2: Real muons (case A \& C) have a pointing track segment both in the inner (EI) and middle (EM) end-cap chambers, while fake triggers (case B) do not. (cfr. [6])

Moreover, given the design limited $p_{T}$ resolution of the trigger detectors, raising the threshold above $20-30 \mathrm{GeV} / \mathrm{c}$ does not effectively reduce the rate but only leads to loss of physics acceptance.

The Level-1 trigger upgrade addresses both the suppression of the fake triggers and improvement in the $p_{T}$ resolution.

For this purpose, for the Phase-I upgrade, a new component, called the New Small Wheel (NSW), will be added in the Level-1 muon trigger, covering the extreme endcap region $(\eta>1.3)$. 
By requiring the additional coincidence of a small wheel segment pointing to the interaction point (IP) and matched to the trigger segment in the big wheel (see Fig. 2), most of the fake triggers may be eliminated. For this to be effective, the required angular resolution has to be comparable with the deviation due to multiple scattering in the calorimeter, which is $2-3 \mathrm{mrad}$ for high $p_{T}$ muons $(>20 \mathrm{GeV} / \mathrm{c})$. Precise angle measurements, with a resolution of $1 \mathrm{mrad}$, can additionally improve the $p_{T}$ resolution in the end-cap region.

During Phase-II, with the corresponding increase of luminosity, the muon rate will be unbearable and a further refinement of the trigger system will be needed. The limited resolution in the barrel and in the outer end-cap big wheel does not allow to enhance the rejection power. Then the possibility to include the information from the precision chambers into the trigger logic is under evaluation; this would allow to achieve the same rejection as the current second-level trigger (a factor of about 10). Even if the long drift time of these detectors makes them not suitable as trigger detector, it may be possible to achieve the goal by using their information with a coarse resolution (even 10 times worsen) from interesting zones of activity pointed by the present trigger detectors.

Similar rejections can be achieved by including the information from the inner tracking detector, as it will be discussed later in section 5 .

\section{The Level-1 Calorimeter Trigger upgrades}

The rates of Level-1 calorimeter trigger will scale as well with luminosity and it would be impossible to stay within the allocated bandwidth as can be seen in Fig. 3.

Recent studies [6] show that, using higher transverse granularity and depth information, a rejection factor of about 3-5 for low $p_{T}$ jets faking electrons can be achieved by implementing shower shape algorithms.

As an example, Fig. 4 shows the distribution of shower shape variables using the 2nd sampling layer of the electromagnetic calorimeters. Further studies on discriminant variables using the 3rd sampling layer and the hadronic calorimeter layers are in progress and could potentially lead to substantial improvements of the energy resolution for $\tau \mathrm{s}$, jets and, more importantly, missing $E_{T}$ triggers.

The additional higher granularity information will require a partial upgrade of both the calorimeter front-end read-out and the trigger system.

The upgrade plan for Phase-I is part of a more general staged program to be implemented over the next decade for the entire HL-LHC lifetime: the ultimate goal is a free-running digital architecture of all individual electromagnetic and hadronic calorimeter channels.

The intermediate stage proposed for Phase-I combines analog and digital trigger read-out, fully compatible with the present analog transmission of the trigger primitives but adding a digital read-out path that contains many of the elements required by the final upgrade.

For example, the electromagnetic calorimeters boards, called Tower Builder Boards (sTBBs), which send trigger primitives to the trigger system, will be replaced to include the digital read-out path. This provides the trigger with finer granularity data in depth and in $\eta$. Alternative designs, such as a fully digital trigger read-out, are also being considered and will be fully evaluated in the next phase of the development: benefits, challenges, risk and cost analysis will be part of a global optimization process of the calorimeter and of its Level-1 trigger system 


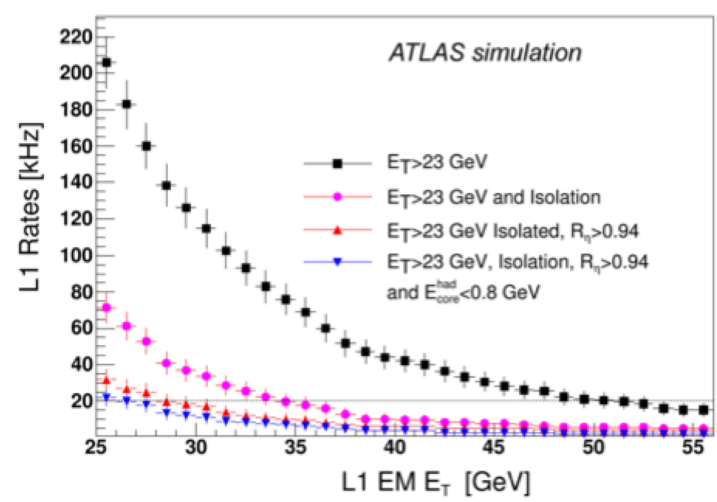

Figure 3: Expected electro-magnetic rates at $\mathscr{L}=$ $10^{34} \mathrm{~cm}^{-2} \mathrm{~s}^{-1}$ as function of the transverse energy threshold for different criteria with the current calorimeter system. (cfr. [6])

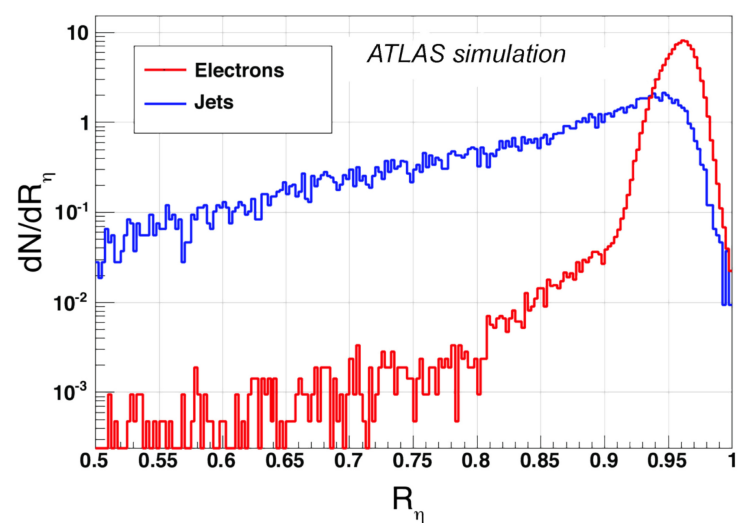

Figure 4: Shape variable ratio $R_{\eta}$ for jets and electrons. The ratio is defined as $R_{\eta}=\frac{E_{3 \times 7}}{E_{7 \times 7}}$ of the energy deposit in a cluster of 3 vs. one of $7 \times 7$ calorimeter cells. (cfr. [6])

For the hadronic calorimeter the full digital read-out is planned for Phase-II. In Phase-I, an upgrade based on using the information of the outermost layer of the hadronic calorimeter, which are already available in the readout is being considered, but a final decision needs to be motivated by further studies.

\section{Level-1 Track Trigger}

During Phase-II the pile-up will substantially impact the calorimeter reconstruction algorithms. For example, the efficiency of calorimeter-based isolation will degrade due to particles from other interaction vertices entering the isolation cones. A Level-1 track system, which is under consideration, can ensure good rejections of fake lepton triggers (electrons, muons and taus) and good b-jet tagging, by requiring the matching of tracks with calorimeter clusters or muons candidates as it is currently done at Level-2.

Moreover, a track-based isolation can use vertex requirements becoming more robust against increased pile-up. This has motivated the installation of a fast Level-2 track trigger [5] for Phase-I.

In Phase-II, the ATLAS inner tracker detector will be replaced by a single all-silicon-detector tracker, with pixel sensors at the inner radii surrounded by microstrip sensors reaching totally order of several hundredths million channels.

While the amount of material needs to be minimized to reduce effects such as photon conversion, energy loss and multiple scattering, on the other hand, it poses limitations to the amount of read-out electronics that can be used. Moreover, given the huge amount of channels, to fully read-out the system a latency of order of few tenth of $\mu$ s would be needed.

It is therefore necessary to filter the data before reading them out of the detector front-ends. The currently proposed scheme splits the Level-1 trigger into a Level-0 and a Level-1 trigger, where the Level-0 specifies regions of the detector to be readout for the Level-1 decision.

The proposed architecture is shown in Fig. 5. The current trigger detectors will work very similarly as today providing the central trigger Level-0 at very high rate and short latency. 
This mechanism would allow to reduce to 5-10\% level the total amount of data to be read-out by the system and then would make it possible the readout of the tracker information within $5 \mu \mathrm{s}$.

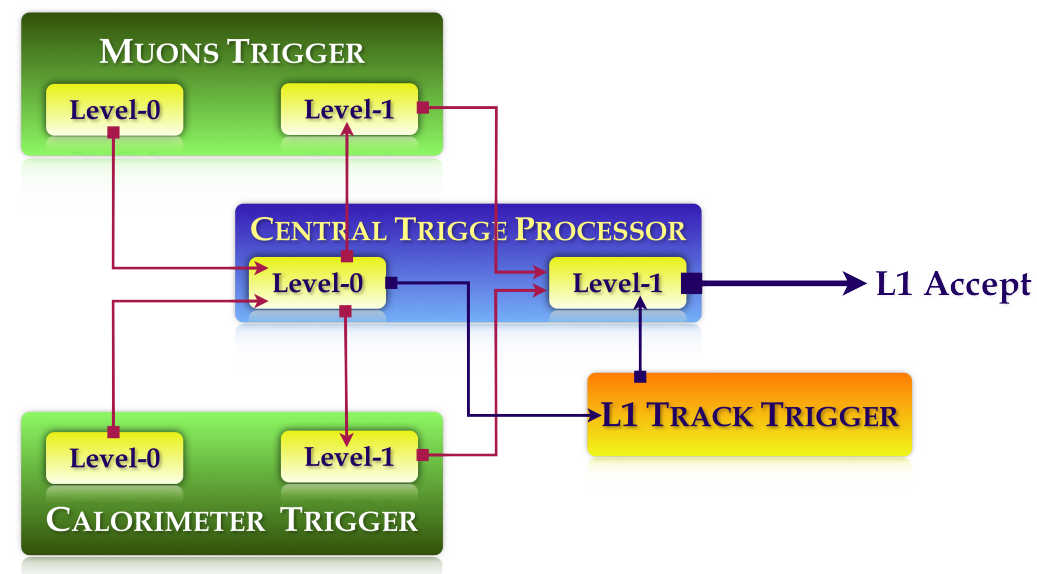

Figure 5: A block scheme of the proposed two-stage Level-1 architecture

\section{Conclusions}

A detailed description of the ATLAS upgrade projects for Phase-I can be found in the dedicated Letter of Intent document [6]. They are already well established and the corresponding Technical Design Reports are under preparation. For the Phase-II upgrade, a Letter of Intent is under preparation and all the Physics studies due to compare different solutions and designs are ongoing. The Letter of Intent for Phase-II should be circulated by early 2013.

\section{References}

[1] J.P. Koutchouk and F. Zimmermann, "LHC Upgrade Scenarios", CERN-sLHC-PROJECT-Report-0013, EuCARD-CON-2009-040, 2010

[2] ATLAS Collaboration, "The ATLAS Experiment at the CERN Large Hadron Collider". JINST, 3:S08003, 2008.

[3] ATLAS Collaboration, "Expected performance of the ATLAS experiment: detector, trigger and physics". ISBN 978-92-9083-321-5

[4] ATLAS Collaboration, "Performance of the ATLAS Trigger System in 2010" The European Physical Journal C - Particles and Fields, DOI:10.1140/epjc/s10052-011-1849-1, 2012

[5] Andreani et al, "The FastTracker real time processor and its impact on muon isolation, tau and b-jet online selections at ATLAS"

IEEE transactions on nuclear science. ISBN: 978-1-4244-7108-9 (2011 Apr), pp. 348-357. DOI: $10.1109 / R T C .2010 .5750337$

[6] ATLAS Collaboration. Letter of intent for the Phase-I upgrade of the atlas experiment. CERN-LHCC-2011-012. LHCC-I-O20, CERN 2011. 